\title{
Uncertainty of mineral prospectivity mapping due to random negative training samples
}

\author{
RENGUANG ZUO*, ZIYE WANG
}

State Key Laboratory of Geological Processes and Mineral Resources, China University of Geosciences, Wuhan 430074, China (*correspondence: zrguang@cug.edu.cn)

This contribution explored the uncertainty of a mineral potential map because of random negative training samples. Both positive and negative training datasets are required for data-driven mineral prospectivity mapping (MPM) Positive training datasets can be generated using the locations of known mineral deposits, and negative training datasets can be created using the locations of random points. However, we cannot confirm that all the negative points are really true negative samples, meaning that there is uncertainty of MPM due to random selection of negative training points. Fifty negative training datasets were generated using the locations of random points, and then 50 mineral potential maps for skarn-type skarn Fe mineralization in southwestern Fujian of China can be obtained by logical regression. We observed that the weight of each evidence layer and the probability of the presence of mineralization for each location within the study area changed in terms of different training datasets, meaning that negative training datasets affected a mineral potential map. The risk and return analysis was used to explore the uncertainty of a mineral potential map due to random negative samples. The mean of the probability obtained through back-transforming the mean of the log odd ratio could be regarded as a final mineral potential map in which the uncertainty due to random negative samples has been considered. The areas having low risk-high return s compared with other locations should be considered firstly for mineral exploration. 